\title{
Extraction of a crack opening from a continuous approach using regularized damage models
}

\author{
Frédéric Dufour ${ }^{1,5}$, Gilles Pijaudier-Cabot ${ }^{1,2}$, Marta Choinska ${ }^{1,3}$ and Antonio Huerta ${ }^{1,4}$ \\ ${ }^{1}$ R\&DO - Institut GeM, Ecole Centrale Nantes, CNRS, France. \\ ${ }^{2}$ Laboratoire des fluides complexes, Université de Pau et des Pays de l'Adour, CNRS, France. \\ ${ }^{3}$ Institut GeM, Université de Nantes, CNRS, France. \\ ${ }^{4}$ Lab. de Càlcul Numèric, Univ. Politècnica de Catalunya, Barcelona, Spain \\ ${ }^{5}$ Corresponding author.
}

\begin{abstract}
Crack opening governs many transfer properties that play a pivotal role in durability analyses. Instead of trying to combine continuum and discrete models in computational analyses, it would be attractive to derive from the continuum approach an estimate of crack opening, without considering the explicit description of a discontinuous displacement field in the computational model. This is the prime objective of this contribution. The derivation is based on the comparison between two continuous variables: the distribution if the effective non local strain that controls damage and an analytical distribution of the effective non local variable that derives from a strong discontinuity analysis. Close to complete failure, these distributions should be very close to each other. Their comparison provides two quantities: the displacement jump across the crack $[U]$ and the distance between the two profiles. This distance is an error indicator defining how close the damage distribution is from that corresponding to a crack surrounded by a fracture process zone. It may subsequently serve in continuous/discrete models in order to define the threshold below which the continuum approach is close enough to the discrete one in order to switch descriptions. The estimation of the crack opening is illustrated on a onedimensional example and the error between the profiles issued from discontinuous and FE analyses is found to be of a few percents close to complete failure.
\end{abstract}




\section{Introduction}

For many concrete structures, crack opening is a key parameter needed in order to estimate durability. Cracks are preferential paths along which fluids or corrosive chemical species may penetrate inside concrete structural elements. For structures such as confinement vessels for instance, tightness to gas or liquids is a major serviceability criterion that is governed by Darcy's relation in which permeability of the material is involved. The material permeability is strongly related to the amount of cracking in concrete: permeability grows significantly as distributed microcracking develops (see e.g. Choinska et al. 2007) and it jumps several orders of magnitude upon macrocracking (Sugyiama et al. 1996, Hearn and Lok, 1998). According to Poiseuille’s law, the permeability of a cracked structure (with a single crack) is proportional to the square of the crack opening. Hence, the prediction of the durability of structural components requires models that describe failure, crack locations and crack openings in the present example too when damage has localised.

- Enhanced continuum and integral damage models are capable of representing diffuse damage, crack initiation and possibly crack propagation (Pijaudier-Cabot and Bazant (1987), Peerlings et al. (1996)). They regard cracking as an ultimate consequence of a gradual loss of material integrity. These models, however, do not predict crack opening as they rely on a continuum approach to fracture.

- Fictitious crack models are based on an explicit description of the discontinuity within the material (e.g. cohesive crack model of Hillerborg et al. (1976)). They relate the crack opening to the stress level and they are based on the linear elastic (or plastic) fracture mechanics. Cohesive crack models needs proper algorithms for crack propagation, and more importantly they are not capable of describing crack initiation.

Ideally, the prediction of durability that involves inception of failure, crack location, propagation and crack opening would require to merge the continuum damage approach and the discrete crack approach into a single, consistent, computational model bridging the continuous and discrete approaches. Bridges between damage and fracture have been devised in the literature (see e.g. Mazars and Pijaudier-Cabot (1996), Planas et al. (1993)). They rely on the equivalence between the dissipation of energy due to damage and the energy dissipated in order to propagate a crack. Given the energy dissipated in the damage process, the equivalent crack length is computed, knowing the fracture energy. Generally, the entire energy that is dissipated in the fracture process zone is "converted" into a crack length (Mazars and Pijaudier-Cabot (1996)). Some part of this energy may be dissipated in the process zone outside from the crack and it follows that the crack length is probably overestimated, and the crack opening is overestimated too. 
The strong discontinuity approach initiated by Simo et al. (1993) and widely used over the last decade (e.g. Oliver et al. (2002), Oliver et al. (2004), Larsson et al. (1998)) offers the possibility of merging in the same formulation a continuous damage model for the bulk response and a cohesive model for the discontinuous part of the kinematics. It is certainly a combination of continuum - discrete modelling that is sound from a theoretical point of view and appealing from the point of view of the physics of fracture. The issue in combining the continuum based model for crack initiation and then a discrete crack model for propagation is, however, the threshold upon which one switches from one analysis to the other. Usually, it is considered that the discontinuity appears when damage, stresses or strain energy reach a certain threshold fixed beforehand, which remains arbitrary (Comi et al. (2007), Simone et al. (2003)). As we will see further, one of the outcome of the present paper is to provide an indicator on the basis of which the appearance of a discontinuity during a damage process can be defined, with a given accuracy.

Instead of trying to combine continuum and discrete models in computational analyses, it would be attractive to derive from the continuum approach an estimate of crack opening, without considering the explicit description of a discontinuous displacement field in the computational model. This derivation could be based on some postprocessing of the distribution of strain and damage in the considered structure. The main purpose of this paper is to present such an estimate of crack opening derived from a continuum model description. First, we recall the continuum approaches that will be considered: the (integral) non local damage model and the gradient damage model. Nonlocal models are known to possess shortcomings such as spurious boundary effects on fracture propagation (Jirasek et al. (2004)) or incorrect initiation of damage at a crack tip (Simone et al. (2004)). Still these defects in the model formulation do not alter their ability to capture a fully localised (mode I) crack. The estimate of crack opening is discussed in the second part. We start from an analytical expression of a regularized strong discontinuity and compare the induced strain field with results from the continuum based model. The comparison of the strain profiles provides the crack opening, and at the same time some indication of the quality of the estimate. Results obtained according to the integral and gradient damage models are illustrated on the one dimensional example of a tension bar. 


\section{II.Non local damage approach}

\section{Damage model}

The scalar isotropic damage model due to Mazars (Mazars and Pijaudier-Cabot (1989)) will be used in the finite element computations for representing the progressive failure. In this model the stress $(\sigma)-$ strain $(\varepsilon)$ relationship is expressed as follows:

$\sigma=(1-D) C: \varepsilon$

where $D$ is the damage scalar variable and $C$ is elastic stiffness of the material. Damage is a combination of two components: $D_{t}$ which is damage due to tension based loads and $D_{c}$ which is damage due to compression:

$D=\alpha_{t} D_{t}+\alpha_{c} D_{c}$

$\alpha_{t}$ and $\alpha_{c}$ depend on the strain tensor. In the case of a uniaxial tensile loading $\alpha_{t}=1$ and $\alpha_{c}=0$. In this paper, we shall be interested damage due to tension loads only, and then Eq. (2) reduces to:

$D=1-\frac{Y_{D 0}\left(1-A_{t}\right)}{Y}-\frac{A_{t}}{e^{\left[B_{t}\left(Y-Y_{D 0}\right)\right]}}$

where $A_{t}, B_{t}$ and $Y_{D 0}$ are model parameters and $Y$ is defined by:

$Y=\max \left(Y, \varepsilon_{e q}\right)$

with $Y=Y_{D 0}$ initially, and the equivalent strain is defined as:

$\varepsilon_{e q}=\sqrt{\sum_{i=1}^{3}\left\langle\varepsilon_{i}\right\rangle_{+}^{2}}$

$\left\langle\varepsilon_{i}\right\rangle_{+}$denotes the positive part of the principal strain $\varepsilon_{i}$.

This constitutive relation exhibits strain softening and two regularization techniques shall be considered in order to avoid mesh dependency and ill-posedness of the governing equations of equilibrium.

\section{Integral non local model}

In the non local damage model, the effective strain (Eq. (5)) is substituted by the average equivalent strain $\bar{\varepsilon}_{e q}$ in the equations governing the growth of damage (Pijaudier-Cabot and Bazant (1987)). The non local average is defined as

$\bar{\varepsilon}_{e q}=\frac{\int_{\Omega} \phi(x-s) \varepsilon_{e q}(s) d s}{\int_{\Omega} \phi(x-s) d s}$ 
Several weight functions exist in the literature, we choose the most often used, i.e. the Gaussian function:

$\phi_{G}(x-s)=\exp \left(\left(\frac{2|x-s|}{l_{c}}\right)^{2}\right)$

where $l_{c}$ is the internal length of the model, a new model parameter compared to the classical - local constitutive model.

\section{Gradient enhanced model}

The second class of regularized model used in this paper follows the same principle, except that the nonlocal variable derives from a Helmholtz equation. The non local equivalent strain $\bar{\varepsilon}_{\text {eq }}$ replaces its local counterpart. It is defined as:

$\bar{\varepsilon}_{e q}=\sqrt{\sum_{i=1}^{3}\left\langle\bar{\varepsilon}_{i}\right\rangle_{+}^{2}}$

where $\overline{\varepsilon_{i}}$ is a regularized principal strain that is solution of the equation

$\bar{\varepsilon}-c \nabla^{2} \bar{\varepsilon}=\varepsilon$

where $\sqrt{c}[\mathrm{~m}]$ provides a characteristic size to the model. Actually, this gradient enhanced regularization technique is a particular case of the integral approach with a specific, Green weight function that can be written in the 1D case (Peerlings et al. (2001)), as:

$\phi_{G F}(\|x-s\|)=\frac{1}{2 l_{c}} \exp \left(-\frac{|x-s|}{l_{c}}\right)$

One can observe that the slope at the centre $(x=s)$ of the Green function $\phi_{G F}$ is finite but non zero, whereas for the Gaussian function $\phi_{G}$ this slope is zero. Therefore, the Green weight function is sharper at the centre, it decreases more rapidly providing less weight for neighbours of point $x$ than the Gaussian weight function.

\section{Estimation of crack opening}

Let us consider the case of a $1 \mathrm{D}$ bar loaded in tension. The bar of length $L$ is clamped at $x=0$ and a constant velocity $v$ is applied at the other end $(x=L)$. We assume that the loading history is such that failure occurs at point $x=x_{0}$ (Fig. 1a) and we look at the ultimate failure state, after the separation of the bar in two pieces, now 
assumed to be free of load. Note that for simplicity, the crack location is assumed to be known in our approach. The displacement field will be constant piecewise with a step at the crack position $x=x_{0}$.

The displacement profile (see Fig. 1b) is described according to a strong discontinuity formulation:

$u_{s d}\left(x, x_{0}\right)=[U] H_{\Gamma}\left(x-x_{0}\right)$

where subscript $s d$ stands for strong discontinuity, $H_{\Gamma}$ is the Heaviside function and $[U]$ is the displacement jump across the crack. From Eq. (11), we may derive the strain field (see Fig. 1c) with the classical symmetric gradient operator $\nabla^{s}$ :

$\varepsilon_{s d}=\nabla^{s} u_{s d}=[U] \delta\left(x-x_{0}\right)$

where $\delta(x)$ is the Dirac function. According to the above regularized damage models, discontinuous displacements may not necessarily exist, or may represent a limit case that is reached upon infinite strain. Hence, trying to compare the strain field in the bar to a discontinuous strain field may be a difficult task. An approximation may be to compare the profiles of the variable that controls the damage growth instead of comparing the strain itself. According to the non local model, this profile is continuous. If it is close to represent a strong discontinuity, then the distribution of the non local effective strain $\bar{\varepsilon}_{e q}\left(x, x_{0}\right)$ should be close to the distribution of effective strain that results from the displacement jump $[U]$.

In order to perform this comparison, an effective strain distribution $\widetilde{\varepsilon}_{s d}\left(x, x_{0}\right)$ and a non local effective strain distribution $\bar{\varepsilon}_{s d}\left(x, x_{0},[U]\right)$ (see Fig. 1d) are computed according to the same procedure as in the non local damage model. 
(a)
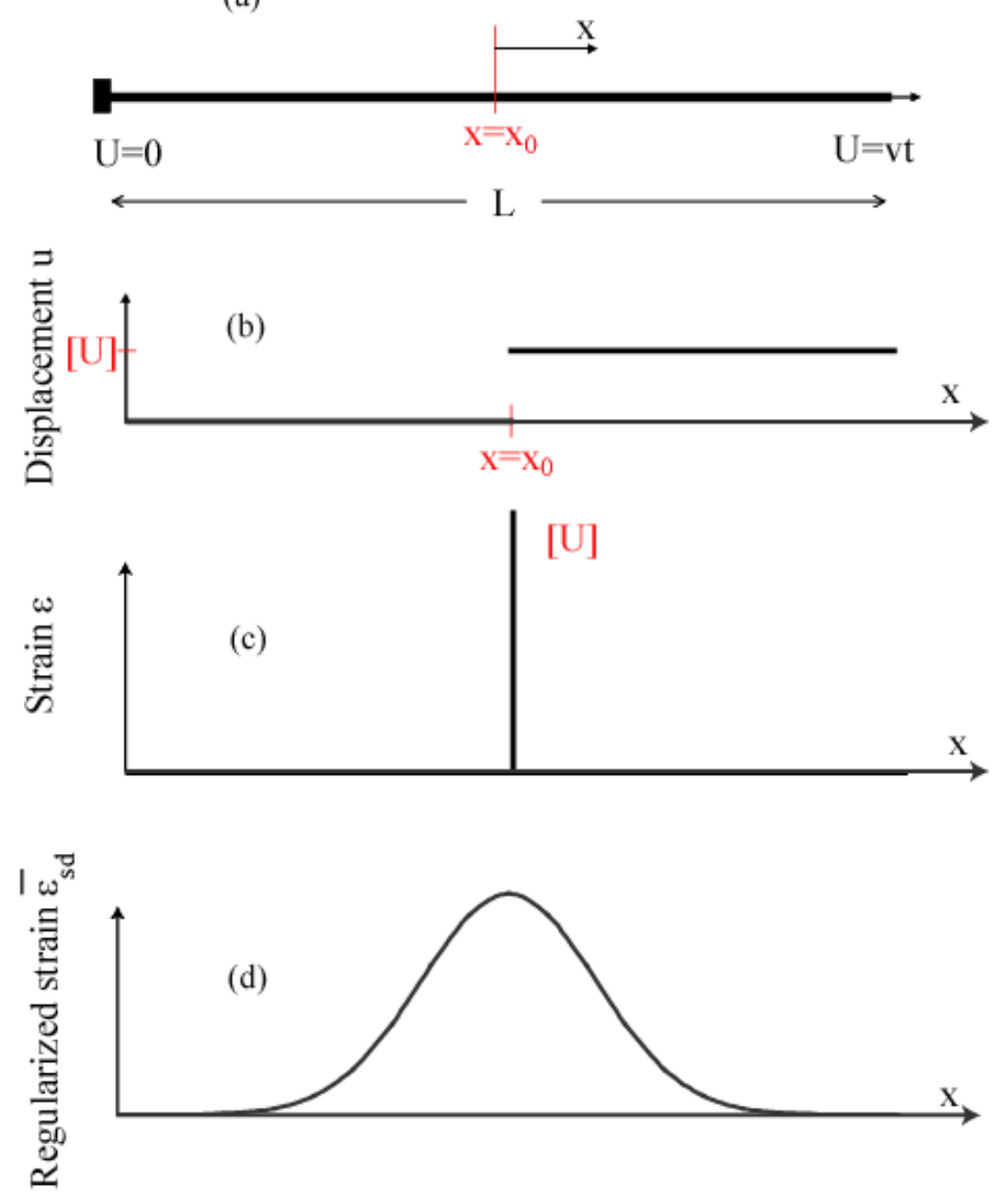

Figure 1: Ultimate state of failure of a 1D bar. (a) Loading system of with a default at $x=x_{0}$ and corresponding displacement field (b), effective strain field (c) and regularized effective strain (d).

$\bar{\varepsilon}_{s d}\left([U], x, x_{0}\right)=\frac{\int_{\Omega} \phi(x-s) \tilde{\varepsilon}_{s d}\left(s, x_{0}\right) d s}{\int_{\Omega} \phi(x-s) d s}$

where $\phi(x)$ is the weight function. Note that in the present case of a $1 \mathrm{D}$ bar, the effective strain $\widetilde{\varepsilon}_{\text {sd }}$ is equal to the strain $\varepsilon_{\text {sd }}$. Substituting Eq. (12) into Eq. (13), and using the properties of the Dirac function, we get:

$\bar{\varepsilon}_{s d}\left([U], x, x_{0}\right)=\frac{[U] \phi\left(x-x_{0}\right)}{\int_{\Omega} \phi(x-s) d s}$

which is a non local measure of the local displacement jump which has the shape of the weighting function. 
The distribution $\bar{\varepsilon}_{e q}\left(x, x_{0}\right)$ is generally obtained numerically whereas $\bar{\varepsilon}_{s d}\left(x, x_{0},[U]\right)$ is known analytically, except for the amplitude of the discontinuity $[U]$. Imposing equality between both regularized effective strains distributions at the location of the crack (which is supposed to be known beforehand), one obtains:

$[U]=\frac{\bar{\varepsilon}_{e q}\left(x_{0}, x_{0}\right) \int_{\Omega} \phi\left(x_{0}-s\right) d s}{\phi(0)}$

and the amplitude of the discontinuity - the crack opening - is derived from this condition (Eq. 15). One could have chosen another condition for computing $[U]$, for instance by minimization of the difference between both profiles. It will not change fundamentally the results if the effective non local distribution is close $\bar{\varepsilon}_{s d}\left(x, x_{0}\right.$, $\left.[U]\right)$. In fact, it should affect the numerical values of the displacement jump when the two nonlocal profiles are not close to each other. In other words, it will influence the values of the jump $[U]$ prior to failure is reached.

Now the distributions $\bar{\varepsilon}_{s d}\left(x, x_{0},[U]\right)$ and $\bar{\varepsilon}_{e q}\left(x, x_{0}\right)$ can be compared and from this comparison, an error indicator can be computed that measures how far from complete failure a given distribution of damage is, and as a consequence the confidence that one may have into the calculation of the displacement jump $[U]$. First, an absolute error field $\Delta$ is defined:

$\Delta\left(x, x_{0},[U]\right)=\bar{\varepsilon}_{s d}\left(x, x_{0},[U]\right)-\bar{\varepsilon}_{e q}\left(x, x_{0}\right)$

Then, an average relative error is evaluated by integrating the absolute error over the bar and normalizing it with the integral of the continuum non local strain:

$$
\Delta^{r}\left(x_{0},[U]\right)=\frac{\int_{\Omega}\left\|\Delta\left(s, x_{0},[U]\right)\right\| d s}{\int_{\Omega} \bar{\varepsilon}_{e q}\left(s, x_{0}\right) d s}
$$

This error indicator defines how close the effective non local strain profile is from that corresponding to a discontinuous displacement distribution. It provides some information on the failure process and at the same time it is an indicator of accuracy of the crack opening estimate: if it is small, it means that complete failure is almost reached, and that the estimate of the crack opening is close to the real displacement discontinuity. This error indicator may subsequently serve in continuous/discrete models in order to define the threshold below which the continuum approach is close enough to the discrete one in order to switch descriptions.

To summarize, we obtain from the comparison of the continuum based non local effective strain and the strong discontinuity based non local effective strain two quantities: (1) the displacement discontinuity (the crack 
opening), and (2) the error associated to the computation of this jump. The error is defined locally at each point of the bar, and globally after some averaging and normalisation. Note that in the absence of localised damage, the error in Eq. (17) is expected to be very high. This is just the consequence that distributed damage is the opposite of localised damage (and cracking) and in the first case, a crack opening does not really exist.

We are going now to apply this scheme to computations performed with the regularized damage models. The integral and gradient damage models will also be compared by means of their capacity to represent the discontinuity jump at failure accurately. In the comparisons, we have set $\phi=\phi_{G}$ in the derivations of the regularized strain fields according to the integral and gradient models. For the gradient model, the weight function is different. It is explicitly known in the $1 \mathrm{D}$ case, but it is not the case in a general 3D calculation. Instead of computing the effective strain distribution that results from the Fredholm equation (Eq. 9) in which the local effective strain is a dirac $\delta$ function, we compute a new average, integral, effective strain from the local strain distribution obtained according to the gradient model with the weight function $\phi=\phi_{G}$. Then, we compare it to the average distribution $\bar{\varepsilon}_{s d}\left(x, x_{0},[U]\right)$ computed with the same weighting function. Therefore, the comparison of effective strains distributions that provide the estimate of crack opening is indirect compared to that in the integral formulation, but again, if the local strain distribution is discontinuous, the two averaged fields should be very close to each other.

\section{Numerical Examples}

We solve numerically the model problem defined in Fig. 1a. The length of the bar is $L=1 \mathrm{~m}$. The location of maximum strain and damage (crack position) is forced in the finite element located at the centre of the bar by setting a defect (smaller Young's modulus $E_{w e}$ ) in this element. Damage will be first triggered in this element by a larger strain than elsewhere. The material parameters of the damage model are provided in Table 1 and Fig. 2 shows the material response in tension.

$$
\begin{array}{cc}
E=37.7 \mathrm{GPa} & Y_{D 0}=10^{-4} \\
E_{w e}=31 \mathrm{GPa} & A_{t}=1 \\
v=0 & B_{t}=20000
\end{array}
$$

Table I. Material parameters for finite element computations. 


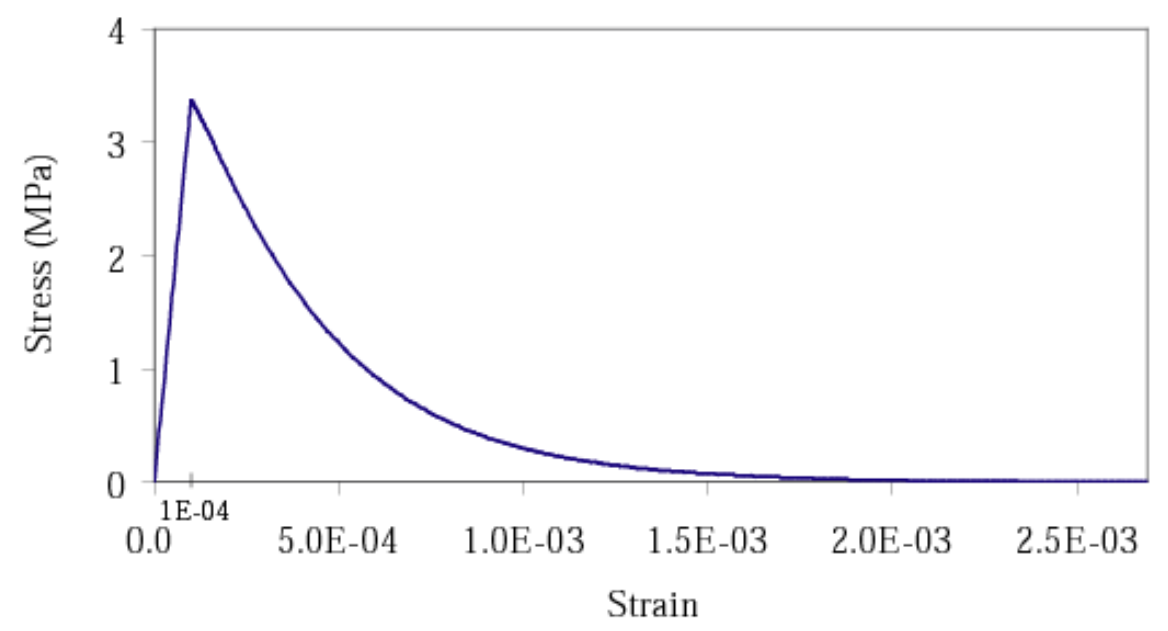

Figure 2: Stress-strain relation for the chosen set of material parameters

In order to exhibit the influence of the internal length and consequently of the size of the fracture process zone (FPZ), we have used two values of the internal length $l_{c}$ in the integral model: $l_{c}=0.18 \mathrm{~m}$ for the small FPZ (SFPZ) and $l_{c}=0.28 m$ for the large FPZ (LFPZ). The $c$ parameter in the gradient enhanced approach was calibrated such that the integral and gradient models give the same FPZ width, that is, the same width of both the damage profiles. We obtained $c=0.005 \mathrm{~m}^{2}$ for the small FPZ and $c=0.012 \mathrm{~m}^{2}$ for the large one. Two finite element meshes are considered too: a coarse mesh of 31 elements and a fine mesh of 61 elements.

\section{Integral and gradient damage responses}

The global response is shown for the gradient model in Fig. 3a and for the non local model in Fig. 3b. These computations have been performed with the smallest value of the internal length in both non local models and for the fine and coarse meshes. We can observe that finite element results are not sensitive on the mesh size, which means that FE convergence with respect to the finite element size has been reached (for both large and small internal lengths). It is worth noting that due to the shape of the weight function (less sharp), the FE convergence is reached for a smaller number of finite element in the case of the integral approach. An arc-length technique has been used to reproduce such a global response which exhibits snap-back. 

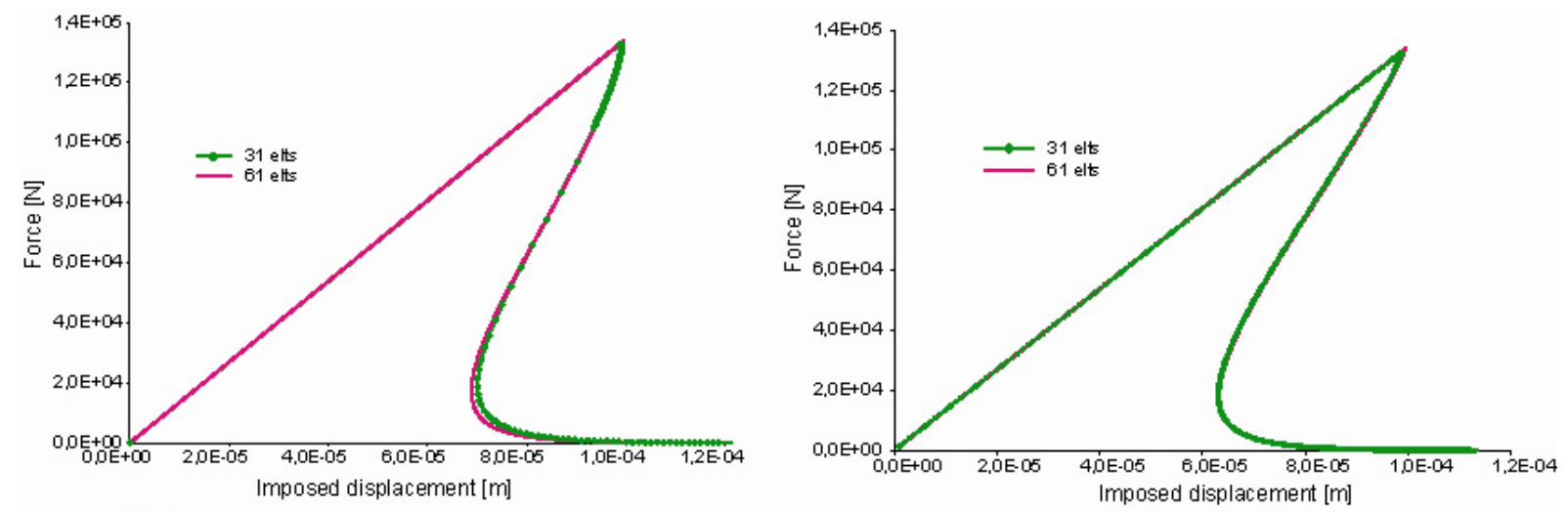

(a)

(c)

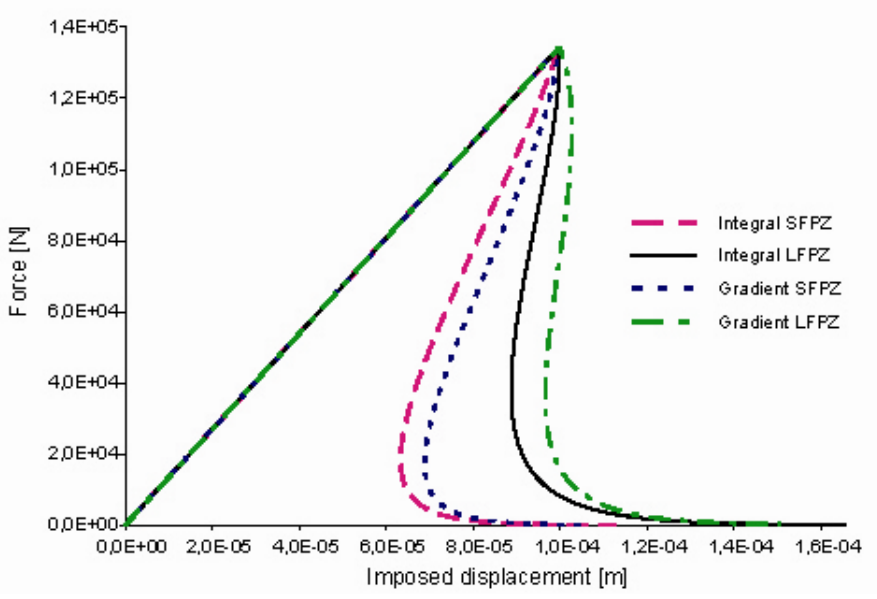

(b)

Figure 3: Global response for the integral approach (a), for the gradient approach (b) for two meshes and for the smallest internal length. The global response (c) for the fine mesh, the two regularization techniques and the two FPZ sizes.

Fig. 3c illustrates the differences between the gradient and integral approaches. Keeping the same size for the FPZ according to both models provides a difference between the load deflection curves. Conversely, Jason et al. (2004) calibrated the gradient model in order to obtain the same load v.s. displacement response as for the integral model. In this case, $l_{c} / \sqrt{c}$ is approximately 2.55 .

We plot damage and strain profiles in the post-peak regime for several ratios of the load to the peak load in the post-peak regime. Fig. 4 shows the corresponding evolution of damage profiles. As soon as damage starts to increase the width of the damaged zone is roughly twice the internal length (of the integral model) and it remains constant throughout the fracture process. Although the FPZ width has been calibrated to be the same for both regularization techniques, one can observe that the gradient model presents sharper peaks on the damage profiles compared to the integral approach. 


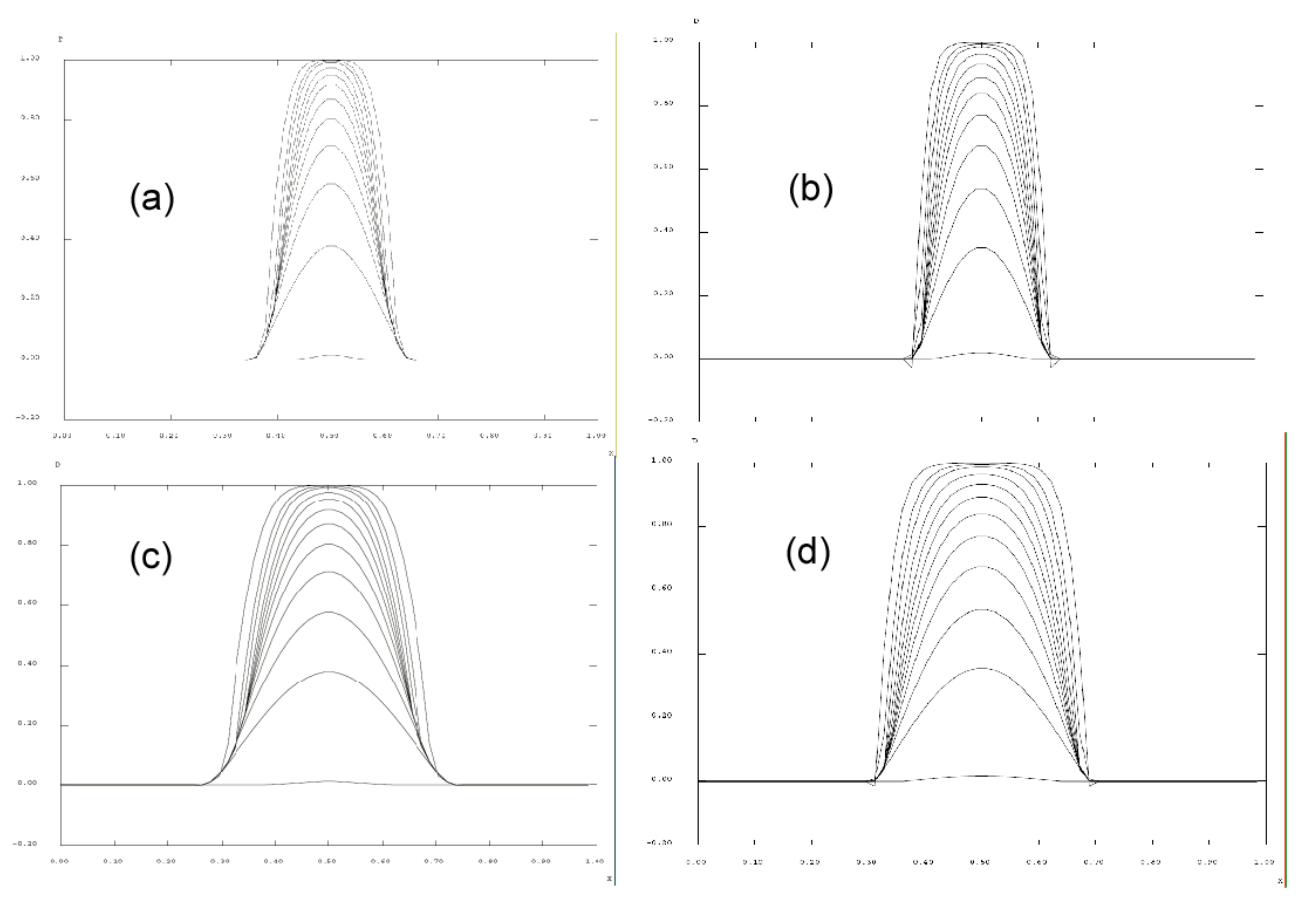

Figure 4: Evolution of damage for the small FPZ with the gradient (a) and the integral (b) approaches, and for the large FPZ with the gradient (c) and the integral (d) approaches. Thick curves corresponds to damage profiles almost at complete failure, when the load is $1 \%$ of the peak load.

Fig. 5 shows the strain profiles along the bar that correspond to the damage profiles in Fig. 4. There is a consistent difference between the integral and the gradient approaches on these curves. The width of the strain profile is smaller according to the gradient approach than that of the integral approach. This is more clearly illustrated with the largest value of the internal length - comparing Fig. 5c and 5d. The strain profile is sharper according to the gradient approach, and this provides the difference observed on the load - displacement responses obtained with the two models. These differences can be regarded also as the consequences of a sharper equivalent weight function according to the gradient approach compared to the Gaussian function used in the integral model. 


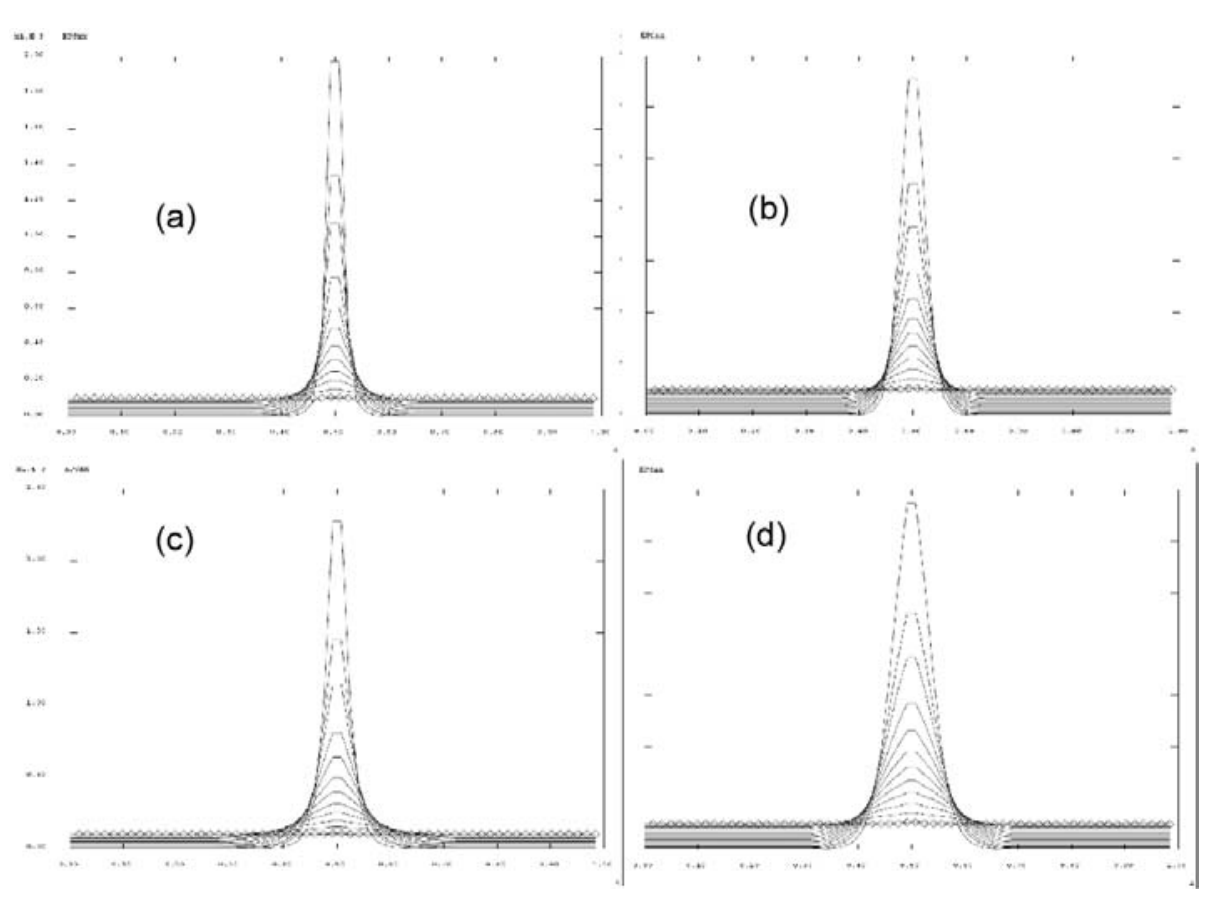

Figure 5: Strain profiles evolution during loading for the small FPZ with the gradient (a) and the integral (b) approaches and for the large FPZ with the gradient (c) and the integral (d) approaches.

\section{Estimation of crack opening}

We are now going to compare the computed regularized effective strain profiles with the profiles derived from the strong discontinuity analysis, derive the estimate of the crack opening and compute the distance between the two profiles which is a failure indicator, or equivalently an error indicator on the accuracy of the crack opening estimate. We consider first a state of damage which is close to complete failure. The ratio of the load to the peak load is 0.01 and the corresponding damage profiles are those drawn with a thick curve in Fig. 4. Figure 6 shows this comparison for the smallest internal length. By definition, the profiles coincide in the centre of the bar, the location with the highest value of damage and effective regularized strain, and the displacement jump $[U]$ is computed from this condition. The profiles deduced from FE computations are wider, but overall, the strong discontinuity profiles and the computed profiles are rather close to each other. 


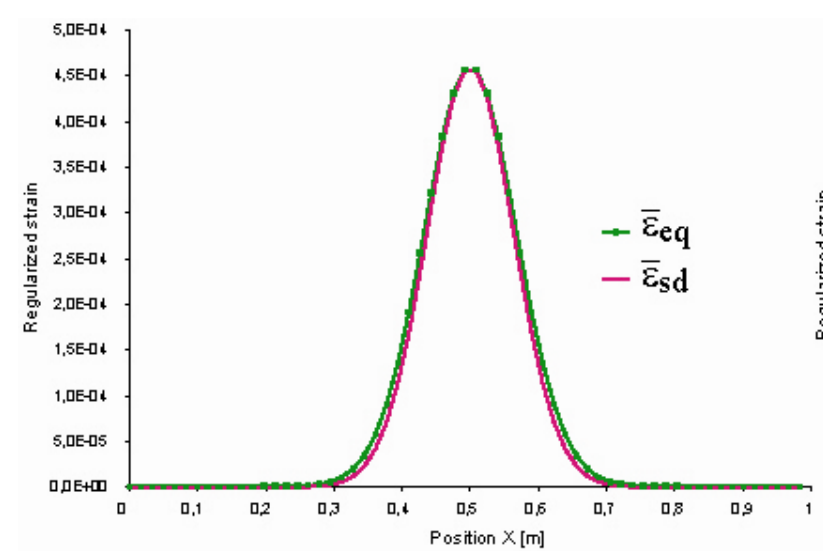

(a)

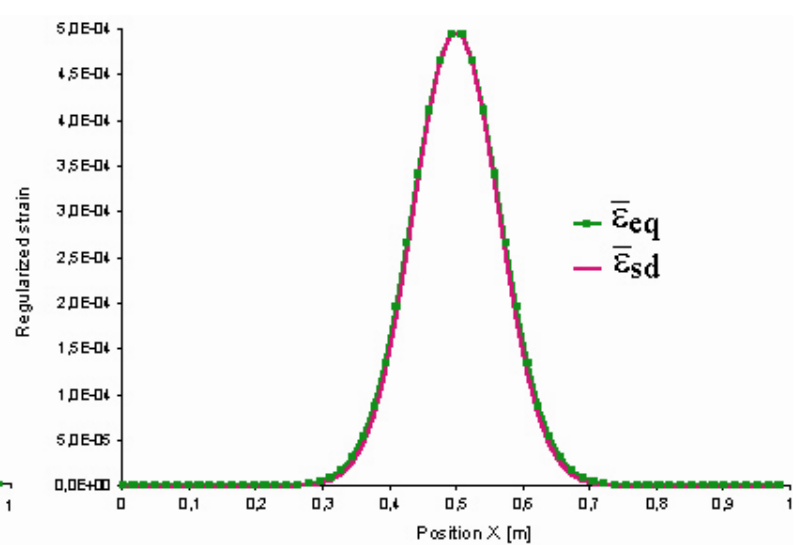

(b)

Figure 6: Regularized effective strain profiles from the strong discontinuity approach (Dirac) and from FE computations using the integral technique (a) or the gradient technique (b) for the smallest FPZ width.

Figure 7 shows the estimates of the crack opening (Fig. 7a) and the evolution of the error indicator computed according to Eq. (17) in the course of the loading history. The load level considered in Fig. 6 corresponds to the black dot in Fig. 7. Once the crack is fully opened the increase of its opening is equal to the displacement applied at the active end of the bar as no further strain are stored in the material (see Fig. 7a). Therefore the plot of the displacement jump versus the imposed displacement tends to the bisecting line. The error indicator (see Fig. 7b) is quite large just after the peak load since the macro-crack is not yet clearly formed. If damage would have been uniformly distributed along the bar, the error would be even higher. Afterwards, the error indicator decreases rapidly to reach a limit value of $4 \%$ for the integral approach and $2 \%$ for the gradient regularization. These values represent the level of ability of such regularized damage models to simulate discontinuous failure in terms of kinematic variables. They illustrate also the influence of the weight function. As pointed out in section 2, the major difference between the gradient and the integral approach is the weight function. With a sharper weight function, the estimate of the crack opening seems to be improved. Still, a limit value of the error of a few percent may be acceptable, provided some validation with experimental data is available. 

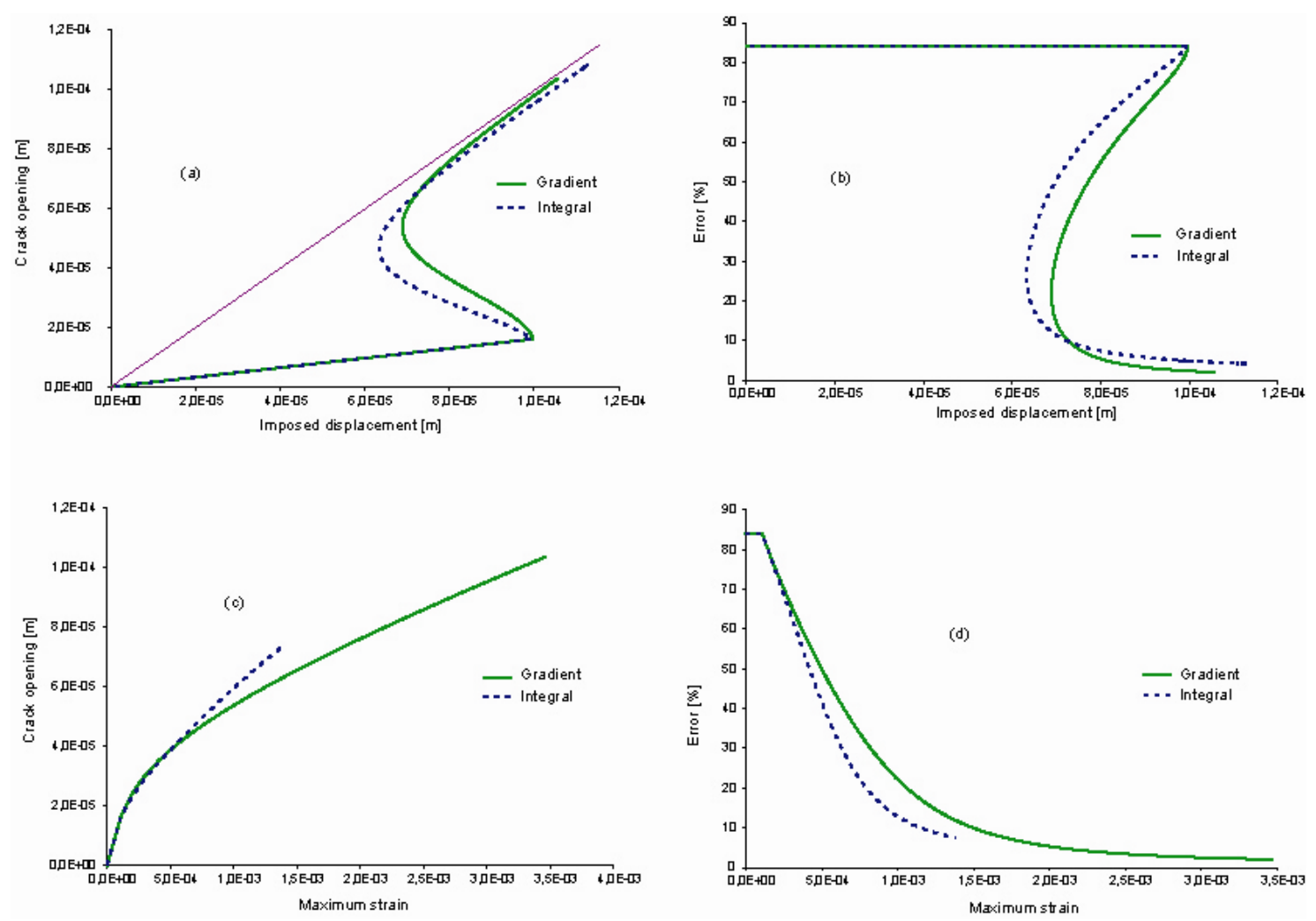

Figure 7: Crack opening as a function of imposed displacement (a) or maximum strain (c) ; relative error as a

function of imposed displacement (b) or maximum strain (d). Plots are given for the two regularization techniques with the smallest FPZ width. The black dot corresponds to a load level of $1 \%$ of the peak load in the softening regime.

Fig. 8 shows that the evolution of the crack opening displacement and the quality of the crack opening estimates are not really dependent on the size of the FPZ. With a small FPZ (small internal length), snap-back on the load displacement response is more severe and limit values of the error are reached for a smaller applied displacement. Nevertheless, the results are quite similar. On this figure, the results obtained with the integral approach have been plotted. Similar observations could be made according to the gradient approach. 

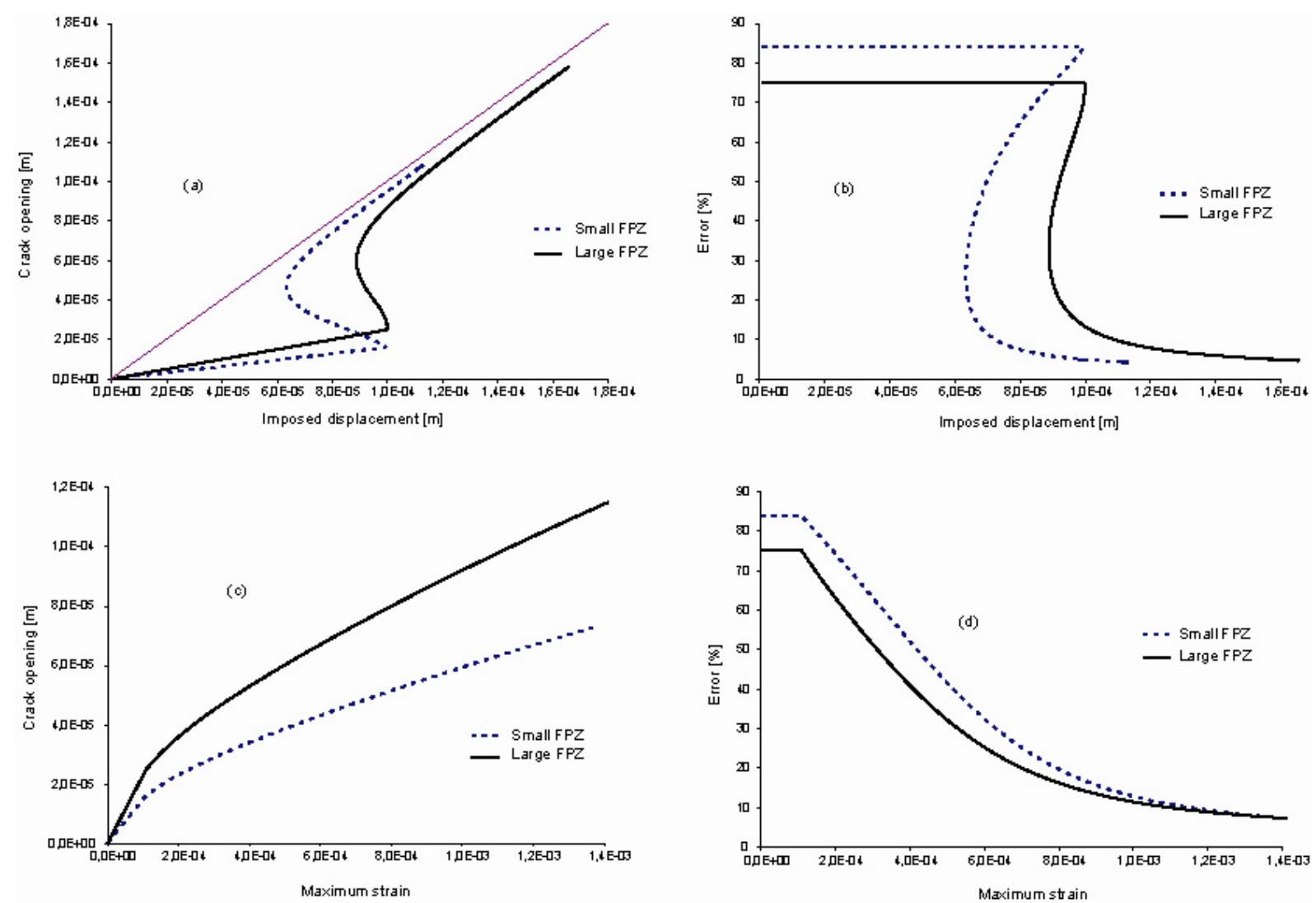

Figure 8: Crack opening as a function of imposed displacement (a) or maximum strain (c); relative error as a function of imposed displacement (b) or maximum strain (d). Plots are given for the integral damage model and for the two sizes of FPZ.

\section{Conclusions}

We have presented in this paper a post processing technique for the evaluation of a crack opening displacement, or its 1D equivalent, a displacement jump, from failure analyses based on regularized damage models. Instead of inserting into the kinematics of the problem the strong discontinuity and the inherent constitutive response, the estimate of the crack opening is based on the comparison of two strain distributions: the regularized effective strain that derives from a non local (integral or gradient) model and the regularized effective strain that is derived from a strong discontinuity that is placed at the expected crack location. By definition, these are two regularized continuous fields that can be approached with standard discretization techniques. If these distributions are close to each other, it means that the variable that control damage has reached a distribution that is close to that of a real crack and it is expected that the corresponding distribution of continuous damage is close to that resulting from a discontinuity (crack) in a regularised, non local, analysis. From the comparison between these two 
regularized fields, the amplitude of the displacement jump and an error indicator on these estimate, that defines also how far from complete failure the distribution of effective strain is, are computed. Close to complete failure, this post processing technique provides an estimate of the crack opening displacement from a finite element computation with an error of a few percent. Although the method is illustrated on a 1D example, it can be extended to 2D analysis too (Legrain et al. (2007)).

The technique applies readily to the integral non local damage model where the regularization is explicit. In the gradient model, the calculation of the regularised strain distribution from the strong discontinuity displacement field is required. An alternative averaging technique - same as for the integral model - has been devised in order to compare effective strain distributions. The comparison between results obtained according to the non local integral and gradient damage models show that both approaches provide results that are similar. The quality of the crack opening estimate depends on the weight function that enters in the non local expression. The gradient approach is equivalent to a non local average with a sharper distribution, lending less weight to neighbouring points compared to the Gaussian distribution in the classical integral approach. As a consequence, the gradient approach provides better limit values of the quality for a formed crack than with the integral model.

Note that when damage is fully developed, the profiles of damage start to enlarge slightly according to the two models. This spurious feature has been already observed by Geers and co-workers (1998) who devised a possible remedy and restricted the non local averaging process to zones where damage grows. This modification of the non local damage model is expected to provide a better description of complete failure and should result into an error indicator on the quality of the crack opening estimate that is improved. This point remains to be investigated, along the quality of the crack opening estimate performed with existing models in which the internal length is changing in the course of damage, and of course validation using experimental data.

Acknowledgement: financial support from the Agence Nationale de la Recherche through the non thematic programme (contract NT05-4_43689) is gratefully acknowledged.

\section{REFERENCES}

Choinska M., Khelidj A., Chatzigeorgiou G., Pijaudier-Cabot G. (2007), Effects and interaction of temperature and stress level related damage on permeability of concrete, Cement and Concrete Research, 37, 79 - 88.

Comi, C., Mariani, S. and Perego, U. (2007). “An extended FE strategy for transition from continuum damage to mode I cohesive crack propagation”, Int. J. for Num. and Anal. Meth. in Geomech., 31(2), 213-238. 
Geers, M.G.D., de Borst, R., Brekelmans, W.A.M., Peerlings, R.H.J., (1998). “Strain-based transient-gradient damage model for failure analyses”, Computer Methods in Applied Mechanics and Engineering, 160, 133-153

Hearn N. and Lok G. (1998), Measurement of Permeability under Uniaxial Compression-A Test Method, ACI Materials Journal, 95, 691-694.

Hillerborg, A., Modeer, M. and Pertersson, P. E. (1976). “Analysis of crack formation and crack growth in concrete by means of fracture mechanics and finite elements”. Cement and Concrete Research, 6, 773-782.

Jason, L., Ghavamian, S., Pijaudier-Cabot, G., Huerta, A., (2004) “Benchmarks for the validation of a non local damage model”, Revue Française de Génie Civil, 8, pp. 303-328.

Jirasek, M., Rosholven, S., Grassl, P. (2004), "Size effect on fracture energy induced by nonlocality”, Int. J. Num. Anal. Meth. Geomechanics, 28, 653-670.

Larsson, R., Steinman, P. and Runesson, K. (1998), “Finite element embedded localization band for finite strain plasticity based on a regularized strong discontinuity”, Mech. of Cohe.-Frict. Mat., 4, 171-194.

Legrain G., Dufour F., Huerta A., Pijaudier-Cabot G. (2007), Extraction of crack opening from a non local damage field, Proceedings of IX International Conference on Computational Plasticity, 1, 462-465, Barcelona, Spain

Mazars, J. and Pijaudier-Cabot, G. (1989), “Continuum damage theory: application to concrete”, J. Engrg. Mech., 115, 345-365.

Mazars, J. and Pijaudier-Cabot, G. (1996), “From damage to fracture mechanics and conversely: a combined approach”, Int. J. of Solids and Struct., 33, 3327-3342.

Oliver, J., Huespe, A. E., Pulido, M. D. G. and Chaves, E. W. V. (2002), "From continuum mechanics to fracture mechanics: the strong discontinuity approach”. Eng. Frac. Mech., 69, 113-136.

Oliver, J., Huespe, A. E., Pulido, M. D. G. and Blanco, S. (2004), “Computational modelling of cracking of concrete in strong discontinuity settings”, Comp. \& Conc., 1, 1, 61-76.

Peerlings, R. H. J., de Borst, R., Brekelmans, W. A. M., de Vree, J. H. P. (1996), “Gradient enhanced damage for quasibrittle materials”, Int. J. Num. Meth. Engrg., 39, 3391-3403.

Peerlings, R. H. J., Geers, M.G.D., de Borst, R., Brekelmans, (2001), “A critical comparison of non local and gradient enhanced softening continua”, Int. J. Solid, Struct., 38, 7723-7746.

Pijaudier-Cabot, G. and Bazant, Z. (1987), “Nonlocal damage theory”, J. of Eng. Mech., 113, 1512-1533.

Planas, J., Elices, M. and Guinea, G. V. (1993), “Cohesive cracks versus nonlocal models: Closing the gap”, Int. J. of Frac., 63, 173-187. 
Simo, J. C., Oliver, J. and Armero, F. (1993), “An analysis of strong discontinuities induced by strain softening in rate-independent inelastic solids”, Comp. Mech., 12, 277-296.

Simone, A., Wells, G. N. and Sluys, L. J. (2003), "From continuous to discontinuous failure in a gradient enhanced continuum damage model”, Comp. Meth. in Appl. Mech. and Eng., 192(41-42), 4581-4607.

Simone, A., Askes, H., Sluys, L.J.,(2004), “Incorrect initiation and propagation of failure in non-local and gradient-enhanced media”, Int. J. Solids Struct. 41, 351-363.

Sugiyama T., Bremner T.W., Holm T.A. (1996), Effect of Stress on Gas Permeability in Concrete, ACI Materials Journal, 93, 443-450.

Keywords: Crack opening, damage model, strong discontinuity, fracture. 